\title{
Use of Allylic Strain to Enforce Stereochemistry. Direct Syntheses of 7,8-Dihydroxycalamenene and Mansonone C
}

George A. Kraus* and Insik Jeon

Department of Chemistry, Iowa State University, Ames, IA 50011

gakraus@iastate.edu

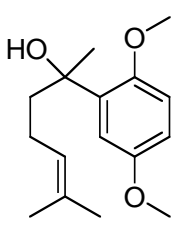

\section{2-(2,5-Dimethoxyphenyl)-6-methylhept-5-en-2-ol}

To a cooled $\left(-78{ }^{\circ} \mathrm{C}\right)$, stirred solution of 1-bromo-2,5-dimethoxybenzene $(1.17 \mathrm{~g}, 10 \mathrm{mmol})$ in THF $(10 \mathrm{~mL})$ was added $n$-BuLi $(4.4 \mathrm{~mL}$ of $2.5 \mathrm{M}$ solution in hexane, $11 \mathrm{mmol})$. The mixture was stirred for $30 \mathrm{~min}$, and then 6-methyl-5-hepten-2-one (1.26 g, $10 \mathrm{mmol})$ in THF ( $5 \mathrm{~mL})$ was added. The resulting mixture was slowly warmed to room temperature, which was then poured into saturated brine. This was extracted with ether $(10 \mathrm{~mL} \times 3)$. The combined organic layers were dried over anhydrous $\mathrm{MgSO}_{4}$ and concentrated in vacuo. The residue was purified by column chromatography on silica gel (EtOAc/hexane, 1:6) to afford the title compound $(2.25 \mathrm{~g}, 85 \%) ;{ }^{1} \mathrm{H} \mathrm{NMR}\left(400 \mathrm{MHz}, \mathrm{CDCl}_{3}\right) \delta 6.92(\mathrm{~d}, J=2.8 \mathrm{~Hz}, 1 \mathrm{H})$, $6.84(\mathrm{~d}, J=8.8 \mathrm{~Hz}, 1 \mathrm{H}), 6.75$ (dd, $J=8.8,2.8 \mathrm{~Hz}, 1 \mathrm{H}), 5.11-5.07$ (m, 1H), 3.85 (s, 3H), 3.78 $(\mathrm{s}, 3 \mathrm{H}), 2.04-1.81(\mathrm{~m}, 4 \mathrm{H}), 1.66(\mathrm{~s}, 3 \mathrm{H}), 1.52(\mathrm{~s}, 3 \mathrm{H}), 1.53(\mathrm{~s}, 3 \mathrm{H}) ;{ }^{13} \mathrm{C}$ NMR $(100 \mathrm{MHz}$, $\left.\mathrm{CDCl}_{3}\right) \delta 153.69,151.08,136.29,131.63,124.66,114.04,112.14,111.47,75.21,55.92$, 55.77, 42.08, 27.46, 25.83, 23.48, 17.71; HRMS (EI) $\mathrm{m} / \mathrm{z}$ calcd for 264.17254, found 264.17286.

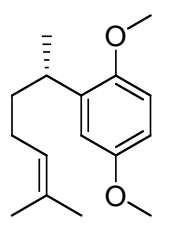

\section{2-(1,5-Dimethylhex-4-enyl)-1,4-dimethoxybenzene}

In a $100 \mathrm{~mL}$, 2-necked, round-bottomed flask fitted with a stirrer, and dry ice condenser were placed 2-(2,5-dimethoxyphenyl)-6-methylhept-5-en-2-ol (1.6 g, $6 \mathrm{mmol}), t$-BuOH $(1.1 \mathrm{~g}, 15 \mathrm{mmol})$, and dry THF $(10 \mathrm{~mL})$. To the cooled $\left(-78^{\circ} \mathrm{C}\right)$, stirred solution $30 \mathrm{~mL}$ of ammonia $\left(\mathrm{NH}_{3}\right)$ was collected and then freshly cut sodium $(0.35 \mathrm{~g}, 15 \mathrm{mmol})$ was added over 3-5 min. The resulting mixture was further stirred until all the sodium was consumed, 
as evidenced by the conversion of the colors to white, and $\mathrm{NH}_{4} \mathrm{Cl}$ powder $(1 \mathrm{~g})$ was added. The temperature of the bath was allowed to rise gradually to room temperature and the reaction was stirred overnight to evaporate ammonia. The resulting mixture was filtered and concentrated in vacuo. After purification by column chromatography (EtOAc/hexane, 1:20), the title compound $(1.36 \mathrm{~g}, 91 \%)$ was isolated; ${ }^{1} \mathrm{H} \mathrm{NMR}\left(400 \mathrm{MHz}, \mathrm{CDCl}_{3}\right) \delta 6.78(\mathrm{~d}$, $J=8.8 \mathrm{~Hz}, 1 \mathrm{H}), 6.77(\mathrm{~d}, J=2.8 \mathrm{~Hz}, 1 \mathrm{H}), 6.68(\mathrm{dd}, J=8.8,2.8 \mathrm{~Hz}, 1 \mathrm{H}), 5.14-5.10(\mathrm{~m}, 1 \mathrm{H})$, $3.78(\mathrm{~s}, 6 \mathrm{H}), 3.19-3.14(\mathrm{~m}, 1 \mathrm{H}), 1.96-1.88(\mathrm{~m}, 2 \mathrm{H}), 1.68(\mathrm{~s}, 3 \mathrm{H}), 1.65-1.50(\mathrm{~m}, 5 \mathrm{H}), 1.19(\mathrm{~d}$, $J=7.2 \mathrm{~Hz}, 3 \mathrm{H}) ;{ }^{13} \mathrm{C} \mathrm{NMR}\left(100 \mathrm{MHz}, \mathrm{CDCl}_{3}\right) \delta 153.86,151.55,137.47,131.25,124.91$, 113.70, 111.56, 110.20, 56.18, 55.67, 37.32, 31.97, 26.39, 25.88, 21.20, 17.74; HRMS (EI) $\mathrm{m} / \mathrm{z}$ calcd for 248.17763 , found 248.17789 .

\section{General procedure for allylic oxidation}

In a $100 \mathrm{~mL}$ round-bottomed flask, $\mathrm{SeO}_{2}(0.5 \mathrm{mmol})$ was dispersed in $\mathrm{CH}_{2} \mathrm{Cl}_{2}(25 \mathrm{~mL})$. $t$ $\mathrm{BuO}_{2} \mathrm{H}(70 \%$ in water, $11 \mathrm{mmol})$ was added dropwise, and the mixture was stirred at room temperature for $10 \mathrm{~min}$. After that time, the mixture was cooled to $0{ }^{\circ} \mathrm{C}$, and then dimethoxybenzenes $(5 \mathrm{mmol})$ in $\mathrm{CH}_{2} \mathrm{Cl}_{2}(10 \mathrm{~mL})$ was slowly added. The reaction was stirred for $36 \mathrm{~h}$, after which time it was poured into $\mathrm{NaOH}(10 \%$ aq.) and extracted with dichloromethane. The organic extracts were washed with water, and then with brine. The solvent was dried over $\mathrm{MgSO}_{4}$, the solids were filtered, and the mixture was then concentrated with the rotary evaporator. The crude was dissolved in methanol $(10 \mathrm{~mL})$ and cooled to $0{ }^{\circ} \mathrm{C}$, and $\mathrm{NaBH}_{4}(5.5 \mathrm{mmol})$ was added to reduce the aldehyde byproduct. After $1 \mathrm{~h}$, the mixture was poured into water, extracted with ether and then washed with brine. The organic extract was dried over $\mathrm{MgSO}_{4}$ and concentrated in vacuo. After evaporation, the residue was purified by column chromatography on silica gel to give the allylic alcohols $(69-71 \%)$.

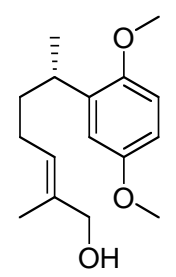

\section{(2E)-6-(2,5-Dimethoxyphenyl)-2-methylhept-2-en-1-ol}

${ }^{1} \mathrm{H}$ NMR $\left(400 \mathrm{MHz}, \mathrm{CDCl}_{3}\right) \delta 6.79(\mathrm{~d}, J=8.8 \mathrm{~Hz}, 1 \mathrm{H}), 6.76(\mathrm{~d}, J=3.2 \mathrm{~Hz}, 1 \mathrm{H}), 6.67$ (dd, $J=8.8,3.2 \mathrm{~Hz}, 1 \mathrm{H}), 5.38(\mathrm{t}, J=7.3 \mathrm{~Hz}, 1 \mathrm{H}), 3.96(\mathrm{~d}, J=6.0 \mathrm{~Hz}, 2 \mathrm{H}), 3.78(\mathrm{~s}, 6 \mathrm{H}), 3.21-3.15$ $(\mathrm{m}, 1 \mathrm{H}), 2.02-1.94(\mathrm{~m}, 2 \mathrm{H}), 1.71-1.57(\mathrm{~m}, 5 \mathrm{H}), 1.19(\mathrm{~d}, J=7.2 \mathrm{~Hz}, 3 \mathrm{H}) ;{ }^{13} \mathrm{C}$ NMR (100 $\left.\mathrm{MHz}, \mathrm{CDCl}_{3}\right) \delta 153.85,151.54,137.25,134.74,126.71,113.78,111.72,110.27,69.22$, $56.34,55.78,36.82,31.86,25.91,21.34,13.75$; HRMS (EI) $\mathrm{m} / \mathrm{z}$ calcd for 264.17254 , found 264.17286. 


\section{General procedure for acetylation}

To a cooled $\left(0{ }^{\circ} \mathrm{C}\right)$, stirred solution of alcohol $(10 \mathrm{mmol})$ in $\mathrm{CH}_{2} \mathrm{Cl}_{2}(30 \mathrm{~mL})$ was added acetic anhydride $(20 \mathrm{mmol})$ and DMAP $(20 \mathrm{mmol})$. The mixture was stirred for $3 \mathrm{~h}$, and then diluted with aq. $\mathrm{NH}_{4} \mathrm{Cl}$. This was extracted with ether $(80 \mathrm{~mL}$ x 2). The combined organic layers were dried over anhydrous $\mathrm{MgSO}_{4}$ and concentrated in vacuo. The residue was purified by column chromatography on silica gel to furnish acetylated compounds (80$91 \%)$.<smiles>COc1ccc(OC)c(C[C@@H](C)CC=C(C)COC(C)=O)c1</smiles>

\section{(2E)-6-(2,5-dimethoxyphenyl)-2-methylhept-2-enyl acetate (8)}

${ }^{1} \mathrm{H}$ NMR $\left(400 \mathrm{MHz}, \mathrm{CDCl}_{3}\right) \delta 6.79(\mathrm{~d}, J=8.8 \mathrm{~Hz}, 1 \mathrm{H}), 6.76(\mathrm{~d}, J=3.2 \mathrm{~Hz}, 1 \mathrm{H}), 6.70(\mathrm{dd}$, $J=8.8,3.2 \mathrm{~Hz}, 1 \mathrm{H}), 5.46(\mathrm{t}, J=6.8 \mathrm{~Hz}, 1 \mathrm{H}), 4.44(\mathrm{~s}, 2 \mathrm{H}), 3.78(\mathrm{~s}, 6 \mathrm{H}), 3.20-3.14(\mathrm{~m}, 1 \mathrm{H})$, 2.07 (s, 2H), 2.06-1.94 (m, 2H), 1.73-1.54 (m, 5H), 1.20 (d, J=7.2 Hz, 3H); ${ }^{13} \mathrm{C}$ NMR (100 $\left.\mathrm{MHz}, \mathrm{CDCl}_{3}\right) \delta 171.12,153.81,151.50,137.01,130.15,129.91,113.71,111.53,110.26$, 70.52, 56.20, 55.72, 36.70, 31.91, 26.04, 21.17, 21.12, 14.01; HRMS (EI) $\mathrm{m} / \mathrm{z}$ calcd for 306.18311 , found 306.18346 .

\section{General procedure for cyclization}

Allylacetate $(0.5 \mathrm{mmol})$ in $1 \mathrm{~mL}$ of TFA/HOAc $(\mathrm{v} / \mathrm{v}=3: 1)$ was stirred at $70{ }^{\circ} \mathrm{C}$ for $12 \mathrm{~h}$. The reaction mixture was quenched with saturated $\mathrm{NaCl}(10 \mathrm{~mL})$ and extracted with ether (15 mL x 3). The combined organic phase was successively washed with water, saturated $\mathrm{NaHCO}_{3}$, and dried over $\mathrm{MgSO}_{4}$, which was then concentrated in vacuo.

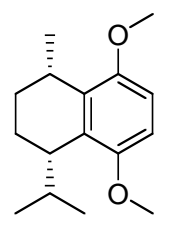

\section{1-Isopropyl-5,8-dimethoxy-4-methyl-1,2,3,4-tetrahydronaphthalene (10)}

Purification by column chromatography on silica gel gave the compound $\mathbf{1 0}$ in $51 \%$ yield; ${ }^{1} \mathrm{H} \mathrm{NMR}\left(400 \mathrm{MHz}, \mathrm{CDCl}_{3}\right) \delta 6.66(\mathrm{~s}, 2 \mathrm{H}), 3.81(\mathrm{~s}, 3 \mathrm{H}), 3.77(\mathrm{~s}, 3 \mathrm{H}), 3.22-3.17(\mathrm{~m}, 1 \mathrm{H})$, 3.03-2.98 (m, 1H), 2.36-2.28 (m, 1H), 1.86-1.75 (m, 2H), 1.65-1.54 (m, 2H), $1.27(\mathrm{~d}, J=7.2$ $\mathrm{Hz}, 3 \mathrm{H}), 0.95(\mathrm{~d}, J=6.8 \mathrm{~Hz}, 3 \mathrm{H}), 0.78(\mathrm{~d}, J=6.8 \mathrm{~Hz}, 3 \mathrm{H}) ;{ }^{13} \mathrm{C} \mathrm{NMR}\left(100 \mathrm{MHz}, \mathrm{CDCl}_{3}\right) \delta$ 151.93, 151.67, 133.32, 131.82, 107.38, 107.17, 55.65, 55.62, 38.48, 30.12, 27.71, 27.26, 21.36, 21.27, 21.15, 18.97; HRMS (EI) $\mathrm{m} / z$ calcd for 248.17763, found 248.17803. 


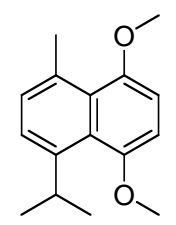

1-Isopropyl-5,8-dimethoxy-4-methylnaphthalene (11)

Purification by column chromatography on silica gel gave the compound $\mathbf{1 1}$ in $37 \%$ yield; ${ }^{1} \mathrm{H}$ NMR $\left(400 \mathrm{MHz}, \mathrm{CDCl}_{3}\right) \delta 7.94(\mathrm{~s}, 1 \mathrm{H}), 7.16(\mathrm{~s}, 1 \mathrm{H}), 6.71-6.66(\mathrm{~m}, 2 \mathrm{H}), 3.97(\mathrm{~s}, 3 \mathrm{H})$, 3.88 (s, 3H), 3.08-2.99 (m, 1H), $2.90(\mathrm{~s}, 3 \mathrm{H}), 1.35$ (d, J=6.8 Hz, 6H); ${ }^{13} \mathrm{C}$ NMR $(100 \mathrm{MHz}$, $\left.\mathrm{CDCl}_{3}\right) \delta 152.44,149.68,146.04,135.13,129.08,128.04,124.66,116.31,104.23,103.51$, $56.00,55.96,34.41,25.33,24.15$; HRMS (EI) $\mathrm{m} / \mathrm{z}$ calcd for 244.14633 , found 244.14671 .

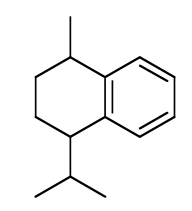

1-Isopropyl-4-methyl-1,2,3,4-tetrahydronaphthalene (13)

Purification by column chromatography on silica gel gave the compound $\mathbf{1 3}$ in $51 \%$ yield as a 1.3:1 mixture of diastereomers; ${ }^{1} \mathrm{H}$ NMR $\left(400 \mathrm{MHz}, \mathrm{CDCl}_{3}\right) \delta 7.26-7.11(\mathrm{~m}, 4 \mathrm{H})$, 2.94-2.92 (m, 0.6H), 2.85-2.80 (m, 0.4H), 2.77-2.72 (m, 0.5H), 2.68-2.63 (m, 0.6H), 2.32$2.21(\mathrm{~m}, 1 \mathrm{H}), 2.03-1.96(\mathrm{~m}, 0.5 \mathrm{H}), 1.90-1.58(\mathrm{~m}, 3 \mathrm{H}), 1.43-1.36(\mathrm{~m}, 0.5 \mathrm{H}), 1.30(\mathrm{~d}, J=7.2$ $\mathrm{Hz}, 1.7 \mathrm{H}), 1.29(\mathrm{~d}, J=7.2 \mathrm{~Hz}, 1.3 \mathrm{H}), 1.05(\mathrm{~d}, J=6.8 \mathrm{~Hz}, 1.7 \mathrm{H}), 1.02(\mathrm{~d}, J=6.8 \mathrm{~Hz}, 1.3 \mathrm{H})$, $0.78(\mathrm{~d}, J=6.8 \mathrm{~Hz}, 1.7 \mathrm{H}), 0.73(\mathrm{~d}, J=7.2 \mathrm{~Hz}, 1.3 \mathrm{H})$; HRMS (EI) $\mathrm{m} / \mathrm{z}$ calcd for 188.15650 , found 188.15684 .

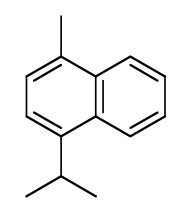

\section{1-Isopropyl-4-methylnaphthalene (14)}

Purification by column chromatography on silica gel gave the compound 14 in $39 \%$ yield; ${ }^{1} \mathrm{H}$ NMR $\left(400 \mathrm{MHz} \mathrm{CDCl}_{3}\right) \delta 8.20-8.17(\mathrm{~m}, 1 \mathrm{H}), 8.07-8.03(\mathrm{~m}, 1 \mathrm{H}), 7.57-7.52(\mathrm{~m}, 2 \mathrm{H})$, $7.33(\mathrm{~d}, J=3.6 \mathrm{~Hz}, 2 \mathrm{H}), 3.80-3.73(\mathrm{~m}, 1 \mathrm{H}), 2.70(\mathrm{~s}, 3 \mathrm{H}), 1.42(\mathrm{~d}, J=6.8 \mathrm{~Hz}, 6 \mathrm{H}) ;{ }^{13} \mathrm{C} \mathrm{NMR}$ $\left(100 \mathrm{MHz}, \mathrm{CDCl}_{3}\right) \delta 133.10,132.24,131.56,126.64,125.51,125.29,125.13,124.00$, $121.55,28.57,23.85,19.74$; HRMS (EI) $\mathrm{m} / \mathrm{z}$ calcd for 184.12520 , found 184.12540 . 


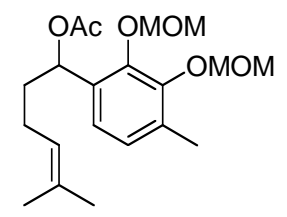

\section{1-[(2,3-Bis(methoxymethoxy)-4-methylphenyl]-5-methylhex-4-enyl acetate}

To a solution of 1,2-bis(methoxymethoxy)-3-methylbenzene (4.3 g, $20 \mathrm{mmol}$ ) in dry THF $(80 \mathrm{~mL})$ was added $n$-BuLi $(8.4 \mathrm{~mL}$ of $2.5 \mathrm{M}$ solution in hexane, $21 \mathrm{mmol})$ at $0{ }^{\circ} \mathrm{C}$. The mixture was stirred for $2 \mathrm{~h}$ at room temperature. The mixture was cooled to $-78{ }^{\circ} \mathrm{C}$, and then 5-methyl-4-hexen-1-al $(2.5 \mathrm{~g}, 22 \mathrm{mmol})$ in THF $(10 \mathrm{~mL})$ was added. The reaction was stirred for additional $1 \mathrm{~h}$ at $-78{ }^{\circ} \mathrm{C}$, and then slowly warmed to room temperature. The resulting mixture was then cooled to $0{ }^{\circ} \mathrm{C}$ and acetic anhydride $(2.1 \mathrm{~mL}, 22 \mathrm{mmol})$ was added. The mixture was stirred for $1 \mathrm{~h}$, and then diluted with brine $(100 \mathrm{~mL})$ and extracted with ether. The combined organic layers were dried over $\mathrm{MgSO}_{4}$, filtered and concentrated under reduced pressure. Flash chromatography on silica gel (EtOAc/hexane, 1:4) yielded the title compound $(5 \mathrm{~g}, 77 \%) ;{ }^{1} \mathrm{H} \mathrm{NMR}\left(400 \mathrm{MHz}, \mathrm{CDCl}_{3}\right) \delta 7.07(\mathrm{~d}, J=8.0 \mathrm{~Hz}, 1 \mathrm{H}), 6.97$ $(\mathrm{d}, J=8.0 \mathrm{~Hz}, 1 \mathrm{H}), 5.19-5.04(\mathrm{~m}, 5 \mathrm{H}), 4.97-4.92(\mathrm{~m}, 1 \mathrm{H}), 3.60(\mathrm{~s}, 3 \mathrm{H}), 3.57(\mathrm{~s}, 3 \mathrm{H}), 2.78(\mathrm{~d}$, $J=4.0 \mathrm{~Hz}, 1 \mathrm{H}), 2.31(\mathrm{~s}, 3 \mathrm{H}), 2.17-2.08(\mathrm{~m}, 2 \mathrm{H}), 1.97-1.88(\mathrm{~m}, 1 \mathrm{H}), 1.83-1.76(\mathrm{~m}, 1 \mathrm{H}), 1.70$ $(\mathrm{s}, 3 \mathrm{H}), 1.61(\mathrm{~s}, 3 \mathrm{H}) ;{ }^{13} \mathrm{C} \mathrm{NMR}\left(100 \mathrm{MHz}, \mathrm{CDCl}_{3}\right) \delta$ 148.27, 148.19, 136.89, 132.18, $132.14,126.86,124.25,122.10$, 99.79, 99.21, 68.14, 57.80, 57.79, 36.61, 25.94, 25.07, 17.88, 16.67; HRMS (EI) $\mathrm{m} / \mathrm{z}$ calcd for 324.19367, found 324.19405 .

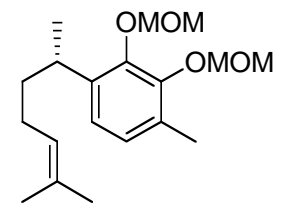

\section{1-(1,5-Dimethylhex-4-enyl)-2,3-bis(methoxymethoxy)-4-methylbenzene (15)}

To a solution of 1-[(2,3-bis(methoxymethoxy)-4-methylphenyl]-5-methylhex-4-enyl acetate $(2.2 \mathrm{~g}, 6 \mathrm{mmol})$ in $\mathrm{CH}_{2} \mathrm{Cl}_{2}(30 \mathrm{~mL})$ was added trimethylaluminum $(9.0 \mathrm{~mL}$ of 2.0 $\mathrm{M}$ solution in hexane, $18 \mathrm{mmol})$ at $-78{ }^{\circ} \mathrm{C}$ under argon. The reaction mixture was warmed to $0{ }^{\circ} \mathrm{C}$ over $3 \mathrm{~h}$, quenched with water, and extracted with ether. The organic layer was washed with saturated aq. $\mathrm{NaHCO}_{3}$, and brine, dried over $\mathrm{MgSO}_{4}$, and evaporated under reduced pressure. The residue was chromatographed on silica gel (EtOAc/hexane, 1:20) to afford the title compound (15) $(1.7 \mathrm{~g}, 88 \%) ;{ }^{1} \mathrm{H} \mathrm{NMR}\left(400 \mathrm{MHz}, \mathrm{CDCl}_{3}\right) \delta 6.92(\mathrm{~d}, J=8.0$ $\mathrm{Hz}, 1 \mathrm{H}), 6.89$ (d, J=8.0 Hz, 1H), 5.13-5.02 (m, 5H), 3.59 (s, 6H), 3.25-3.19 (m, 1H), 2.29 $(\mathrm{s}, 3 \mathrm{H}), 2.02-1.83(\mathrm{~m}, 2 \mathrm{H}), 1.76-1.55(\mathrm{~m}, 8 \mathrm{H}), 1.19(\mathrm{~d}, J=7.2 \mathrm{~Hz}, 3 \mathrm{H}) ;{ }^{13} \mathrm{C}$ NMR $(100 \mathrm{MHz}$, $\left.\mathrm{CDCl}_{3}\right) \delta 148.50,147.60,140.06,129.90,126.91,126.49,124.79,121.92,99.50,99.29$, 57.74, 57.68, 38.04, 31.39, 26.43, 25.87, 22.00, 17.80, 16.57; HRMS (EI) $\mathrm{m} / z$ calcd for 322.21441 , found 322.21491 .

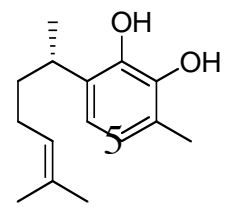




\section{3-(1,5-Dimethylhex-4-enyl)-6-methylbenzene-1,2-diol}

To a slution of 1-(1,5-dimethylhex-4-enyl)-2,3-bis(methoxymethoxy)-4-methylbenzene $(161 \mathrm{mg}, 0.5 \mathrm{mmol})$ in $i$-PrOH $(1.6 \mathrm{~mL})$ was added $\mathrm{AcCl}(0.18 \mathrm{~mL}, 2.5 \mathrm{mmol})$ at $0^{\circ} \mathrm{C}$. The reaction was monitored by TLC to avoid prolonged reaction times. After completion (5h), $\mathrm{H}_{2} \mathrm{O}(15 \mathrm{~mL})$ was added and the mixture was extracted with EtOAc $(3 \times 20 \mathrm{~mL})$. The combined organic phases were washed with $\mathrm{H}_{2} \mathrm{O}$ and concentrated to afford quantitative yield of the title compound, which is used for the next step without further purification; ${ }^{1} \mathrm{H}$ NMR $\left(400 \mathrm{MHz}, \mathrm{CDCl}_{3}\right) \delta 6.71(\mathrm{~d}, J=8.0 \mathrm{~Hz}, 1 \mathrm{H}), 6.67(\mathrm{~d}, J=8.0 \mathrm{~Hz}, 1 \mathrm{H}), 5.25-5.17(\mathrm{~m}$, $3 \mathrm{H}), 3.01-2.95(\mathrm{~m}, 1 \mathrm{H}), 2.25(\mathrm{~s}, 3 \mathrm{H}), 1.99-1.94(\mathrm{~m}, 2 \mathrm{H}), 1.73(\mathrm{~s}, 3 \mathrm{H}), 1.70-1.63(\mathrm{~m}, 2 \mathrm{H})$, $1.57(\mathrm{~s}, 3 \mathrm{H}), 1.27$ (d, J=7.2 Hz, 3H); $\left.{ }^{13} \mathrm{C} \mathrm{NMR} \mathrm{(100} \mathrm{MHz,} \mathrm{CDCl}_{3}\right) \delta$ 142.07, 141.12, 132.74, 131.24, 124.97, 122.43, 121.31, 117.92, 37.59, 31.73, 26.16, 25.94, 21.41, 17.94, 15.57; HRMS (EI) $m / z$ calcd for 262.19328, found 262.19376.

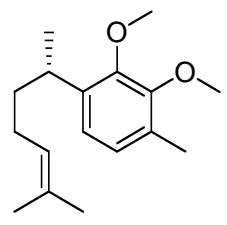

\section{1-(1,5-Dimethylhex-4-enyl)-2,3-dimethoxy-4-methylbenzene (16)}

To a slution of 3-(1,5-dimethylhex-4-enyl)-6-methylbenzene-1,2-diol (117 mg, $0.5 \mathrm{mmol})$ in DMF (3 mL) was added $\mathrm{NaH}(60 \mathrm{mg}$ of $60 \%$ dispersed in mineral oil, $1.5 \mathrm{mmol})$, and the solution was stirred at room temperature for $30 \mathrm{~min}$. After which MeI $(213 \mathrm{mg}, 1.5$ mmol) was added and the reaction was stirred overnight. It was then diluted with aq. $\mathrm{NH}_{4} \mathrm{Cl}$. This was extracted with ether $(10 \mathrm{~mL}$ x 2). The combined organic layers were dried over anhydrous $\mathrm{MgSO}_{4}$ and concentrated in vacuo. The residue was purified by column chromatography on silica gel to furnish the title compound (16) $(125 \mathrm{mg}, 95 \%)$; ${ }^{1} \mathrm{H} \mathrm{NMR}$ $\left(400 \mathrm{MHz}, \mathrm{CDCl}_{3}\right) \delta 6.71(\mathrm{~d}, J=8.0 \mathrm{~Hz}, 1 \mathrm{H}), 6.67(\mathrm{~d}, J=8.0 \mathrm{~Hz}, 1 \mathrm{H}), 5.25-5.17(\mathrm{~m}, 3 \mathrm{H})$, 3.01-2.95 (m, 1H), $2.25(\mathrm{~s}, 3 \mathrm{H}), 1.99-1.94(\mathrm{~m}, 2 \mathrm{H}), 1.73(\mathrm{~s}, 3 \mathrm{H}), 1.70-1.63(\mathrm{~m}, 2 \mathrm{H}), 1.57(\mathrm{~s}$, $3 \mathrm{H}), 1.27(\mathrm{~d}, J=7.2 \mathrm{~Hz}, 3 \mathrm{H}) ;{ }^{13} \mathrm{C} \mathrm{NMR}\left(100 \mathrm{MHz}, \mathrm{CDCl}_{3}\right) \delta$ 142.07, 141.12, 132.74, 131.24, 124.97, 122.43, 121.31, 117.92, 37.59, 31.73, 26.16, 25.94, 21.41, 17.94, 15.57; HRMS (EI) $\mathrm{m} / \mathrm{z}$, calcd for 262.19328, found 262.19376. 


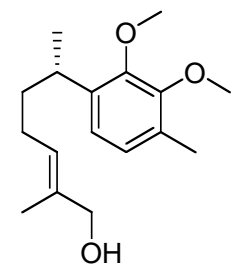

(2E)-6-(2,3-Dimethoxy-4-methylphenyl)-2-methylhept-2-en-1-ol

${ }^{1} \mathrm{H}$ NMR $\left(400 \mathrm{MHz}, \mathrm{CDCl}_{3}\right) \delta 6.89(\mathrm{~d}, J=8.0 \mathrm{~Hz}, 1 \mathrm{H}), 6.83(\mathrm{~d}, J=8.0 \mathrm{~Hz}, 1 \mathrm{H}), 5.39(\mathrm{t}$, $J=7.2 \mathrm{~Hz}, 1 \mathrm{H}), 4.01(\mathrm{~s}, 2 \mathrm{H}), 3.84(\mathrm{~s}, 3 \mathrm{H}), 3.83(\mathrm{~s}, 3 \mathrm{H}), 3.20-3.11(\mathrm{~m}, 1 \mathrm{H}), 2.25(\mathrm{~s}, 3 \mathrm{H})$, 2.05-1.89 (m, 2H), 1.71-1.58 (m, 2H), $1.56(\mathrm{~s}, 3 \mathrm{H}), 1.20(\mathrm{~d}, J=6.8 \mathrm{~Hz}, 3 \mathrm{H}) ;{ }^{13} \mathrm{C}$ NMR $(100$ $\left.\mathrm{MHz}, \mathrm{CDCl}_{3}\right) \delta 151.40,150.87,139.05,134.86,129.62,126.68,125.87,121.62,69.25$, $60.90,60.09,37.36,31.64,26.04,22.48,15.86,13.82$; HRMS (EI) $\mathrm{m} / \mathrm{z}$ calcd for 278.18819 , found 278.18867 .

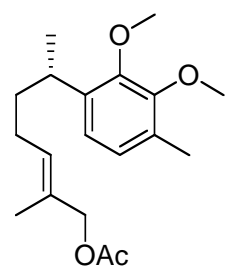

(2E)-6-(2,3-dimethoxy-4-methylphenyl)-2-methylhept-2-enyl acetate (17)

${ }^{1} \mathrm{H}$ NMR $\left(400 \mathrm{MHz}, \mathrm{CDCl}_{3}\right) \delta 6.88(\mathrm{~d}, J=8.0 \mathrm{~Hz}, 1 \mathrm{H}), 6.83(\mathrm{~d}, J=8.0 \mathrm{~Hz}, 1 \mathrm{H}), 5.47(\mathrm{t}$, $J=7.2 \mathrm{~Hz}, 1 \mathrm{H}), 4.44(\mathrm{~s}, 2 \mathrm{H}), 3.84(\mathrm{~s}, 3 \mathrm{H}), 3.83(\mathrm{~s}, 3 \mathrm{H}), 3.17-3.12(\mathrm{~m}, 1 \mathrm{H}), 2.25(\mathrm{~s}, 3 \mathrm{H})$, $2.07(\mathrm{~s}, 3 \mathrm{H}), 2.06-1.91(\mathrm{~m}, 2 \mathrm{H}), 1.70-1.55(\mathrm{~m}, 5 \mathrm{H}), 1.21(\mathrm{~d}, J=7.2 \mathrm{~Hz}, 3 \mathrm{H}) ;{ }^{13} \mathrm{C}$ NMR $(100$ $\left.\mathrm{MHz}, \mathrm{CDCl}_{3}\right) \delta 171.10,151.39,150.92,138.95,130.05,130.00,129.62,125.78,121.51$, $70.48,60.76,60.01,37.32,31.83,26.24,22.15,21.16,15.82,14.06$; HRMS (EI) $\mathrm{m} / z$ calcd for 320.19876 , found 320.19919 .

\section{Procedure for demethylation reaction}

After cyclization reaction of 17, Allylacetate $(0.5 \mathrm{mmol})$ in $1 \mathrm{~mL}$ of TFA/HOAc $(\mathrm{v} / \mathrm{v}=$ 3:1) was stirred at $70{ }^{\circ} \mathrm{C}$ for $12 \mathrm{~h}$. The reaction mixture was quenched with saturated $\mathrm{NaCl}$ $(10 \mathrm{~mL})$ and extracted with ether $(15 \mathrm{~mL} \times 3)$. The combined organic phase was successively washed with water, saturated $\mathrm{NaHCO}_{3}$, and dried over $\mathrm{MgSO}_{4}$. After evaporation, the residue was dissolved in $\mathrm{CH}_{2} \mathrm{Cl}_{2}$ and cooled to $-78{ }^{\circ} \mathrm{C}$ with stirring. To this solution, $\mathrm{BBr}_{3}\left(1 \mathrm{M}\right.$ solution in $\mathrm{CH}_{2} \mathrm{Cl}_{2}, 1 \mathrm{mmol}$ ) was added and stirred for $1 \mathrm{~h}$. Meanwhile, the reaction mixture was slowly warmed to room temperature. After this, aq. $10 \% \mathrm{NaOH}$ solution $(2 \mathrm{~mL})$ was added. The resulting hydrolyzed mixture was then acidified with aq. $10 \% \mathrm{HCl}$ and extracted with ether. The combined organic layers were dried over anhydrous $\mathrm{MgSO}_{4}$ and concentrated in vacuo. 


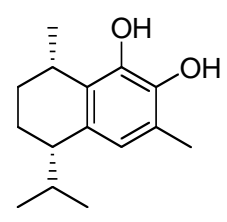

7,8-Dihydroxycalamenene (1a)

Purification by column chromatography on silica gel gave the compound 1a in two steps $30 \%$ yield; ${ }^{1} \mathrm{H}$ NMR $\left(400 \mathrm{MHz}, \mathrm{CDCl}_{3}\right) \delta 6.64(\mathrm{~s}, 1 \mathrm{H}), 5.09(\mathrm{~s}, 1 \mathrm{H}), 4.74(\mathrm{~s}, 1 \mathrm{H}), 3.08-3.04$ $(\mathrm{m}, 1 \mathrm{H}), 2.68-2.62(\mathrm{~m}, 1 \mathrm{H}), 2.39-2.32(\mathrm{~m}, 1 \mathrm{H}), 2.23(\mathrm{~s}, 3 \mathrm{H}), 1.78-1.61(\mathrm{~m}, 4 \mathrm{H}), 1.26(\mathrm{~d}$, $J=7.2 \mathrm{~Hz}, 3 \mathrm{H}), 1.04(\mathrm{~d}, J=6.8 \mathrm{~Hz}, 3 \mathrm{H}), 0.67$ (d, J=6.8 Hz, 3H); ${ }^{13} \mathrm{C}$ NMR $(100 \mathrm{MHz}$, $\left.\mathrm{CDCl}_{3}\right) \delta 141.04,139.04,132.37,127.84,121.26,121.00,42.95,30.87,29.02,27.22,21.25$, 20.72, 17.52, 16.54, 15.87; HRMS (EI) $\mathrm{m} / z$ calcd for 234.16198, found 234.16227.

\section{Mansonone C (2)}

Purification by column chromatography on silica gel gave mansonone $\mathrm{C}(2)$ in two steps $46 \%$ yield as an orange solid; mp $134-137{ }^{\circ} \mathrm{C} ;{ }^{1} \mathrm{H}$ NMR $(400 \mathrm{MHz}, \mathrm{CDCl} 3) \delta 7.66$ (s, 1H), $7.44(\mathrm{~d}, \mathrm{~J}=8.0 \mathrm{~Hz}, 1 \mathrm{H}), 7.20(\mathrm{~d}, \mathrm{~J}=8.0 \mathrm{~Hz}, 1 \mathrm{H}), 3.43-3.36(\mathrm{~m}, 1 \mathrm{H}), 2.64(\mathrm{~s}, 3 \mathrm{H}), 2.09$ (s, $3 \mathrm{H}), 1.30(\mathrm{~d}, J=6.8 \mathrm{~Hz}, 6 \mathrm{H}) ;{ }^{13} \mathrm{C}$ NMR $\left(100 \mathrm{MHz}, \mathrm{CDCl}_{3}\right) \delta 182.49,182.18,145.51$, 143.24, 138.23, 135.18, 134.34, 132.63, 132.16, 129.46, 28.49, 23.97, 23.13, 16.25; HRMS (EI) $\mathrm{m} / \mathrm{z}$ calcd for 228.11503 , found 228.11537. 


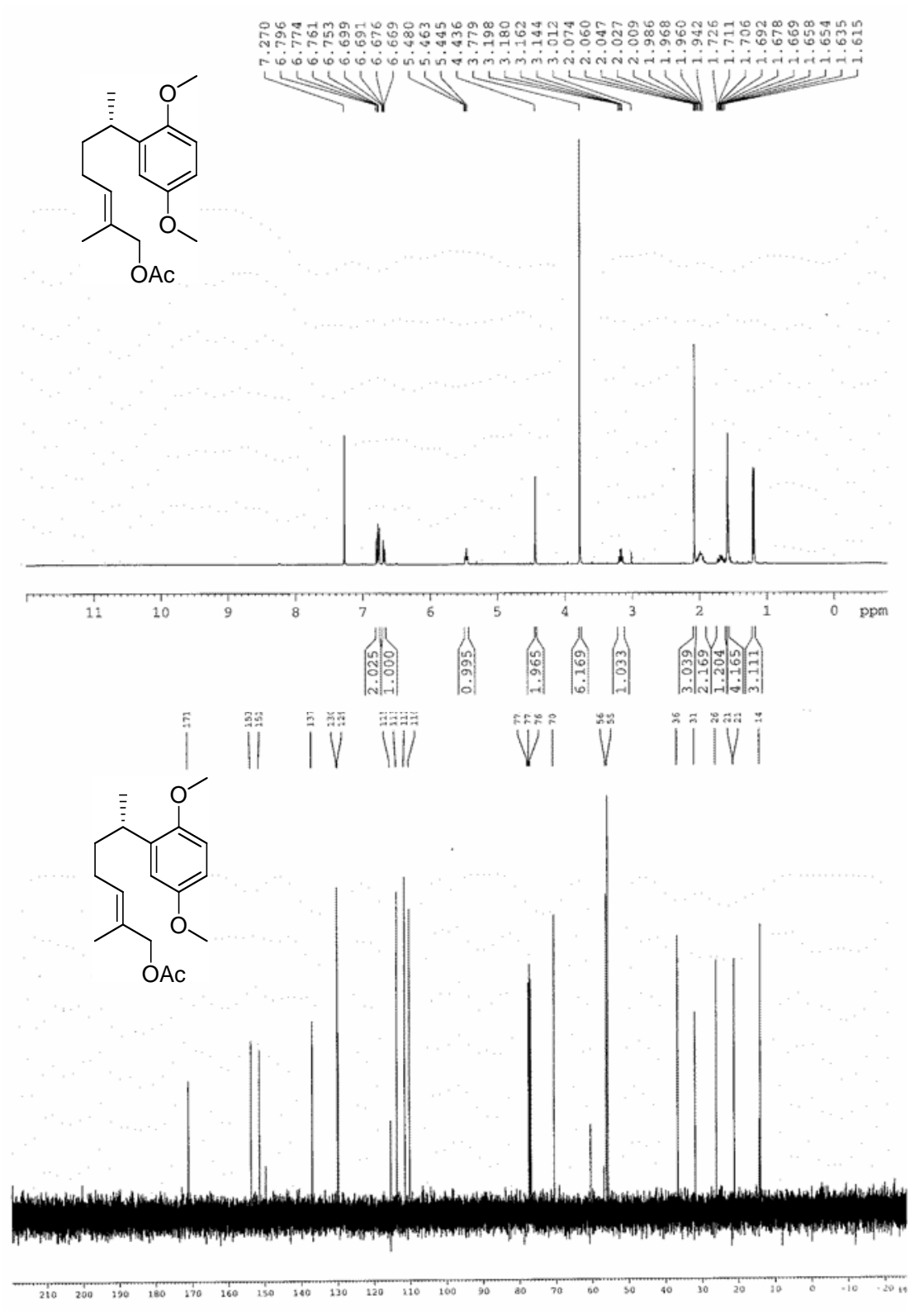

(2E)-6-(2,5-dimethoxyphenyl)-2-methylhept-2-enyl acetate (8) 

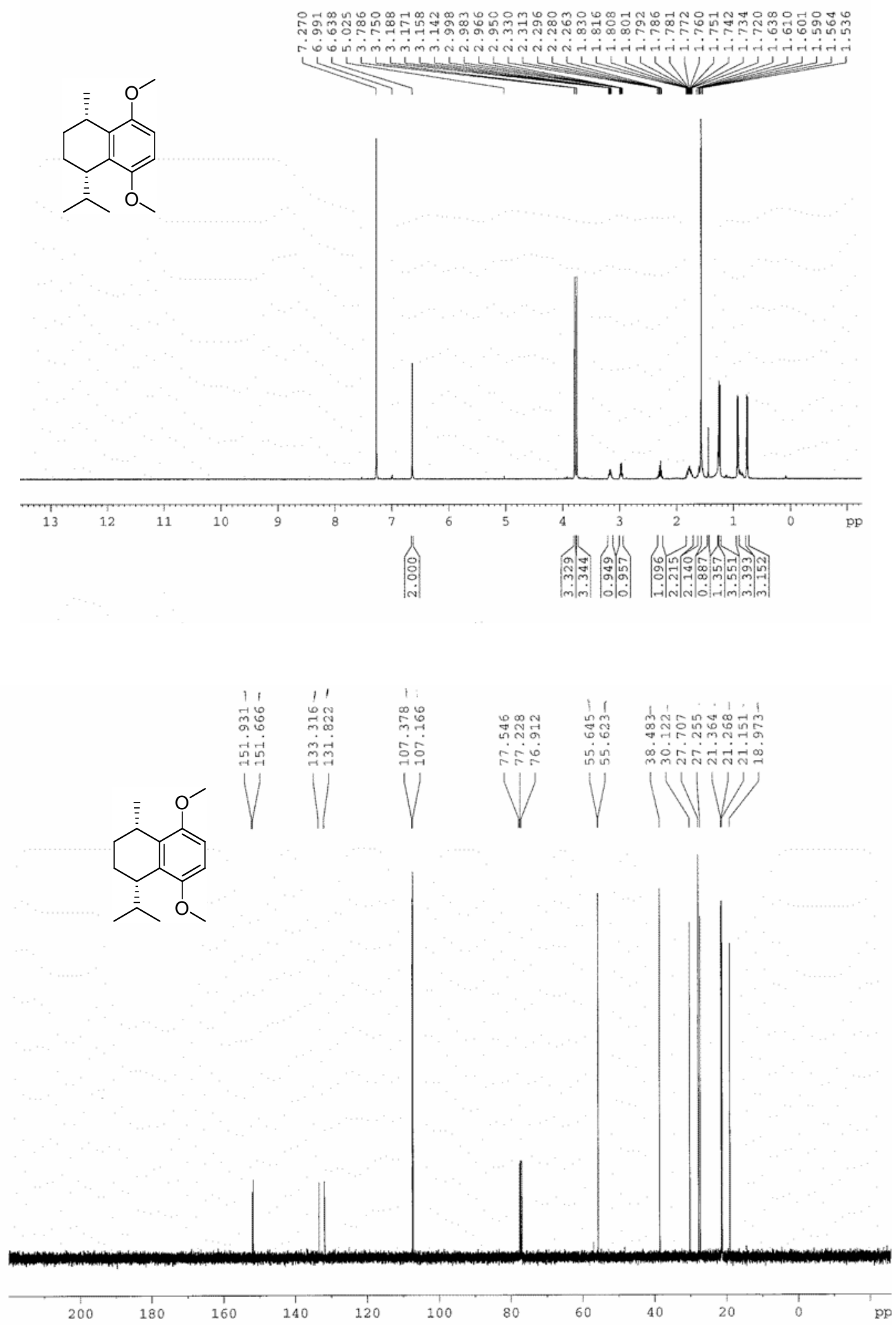

1-Isopropyl-5,8-dimethoxy-4-methyl-1,2,3,4-tetrahydronaphthalene (10) 


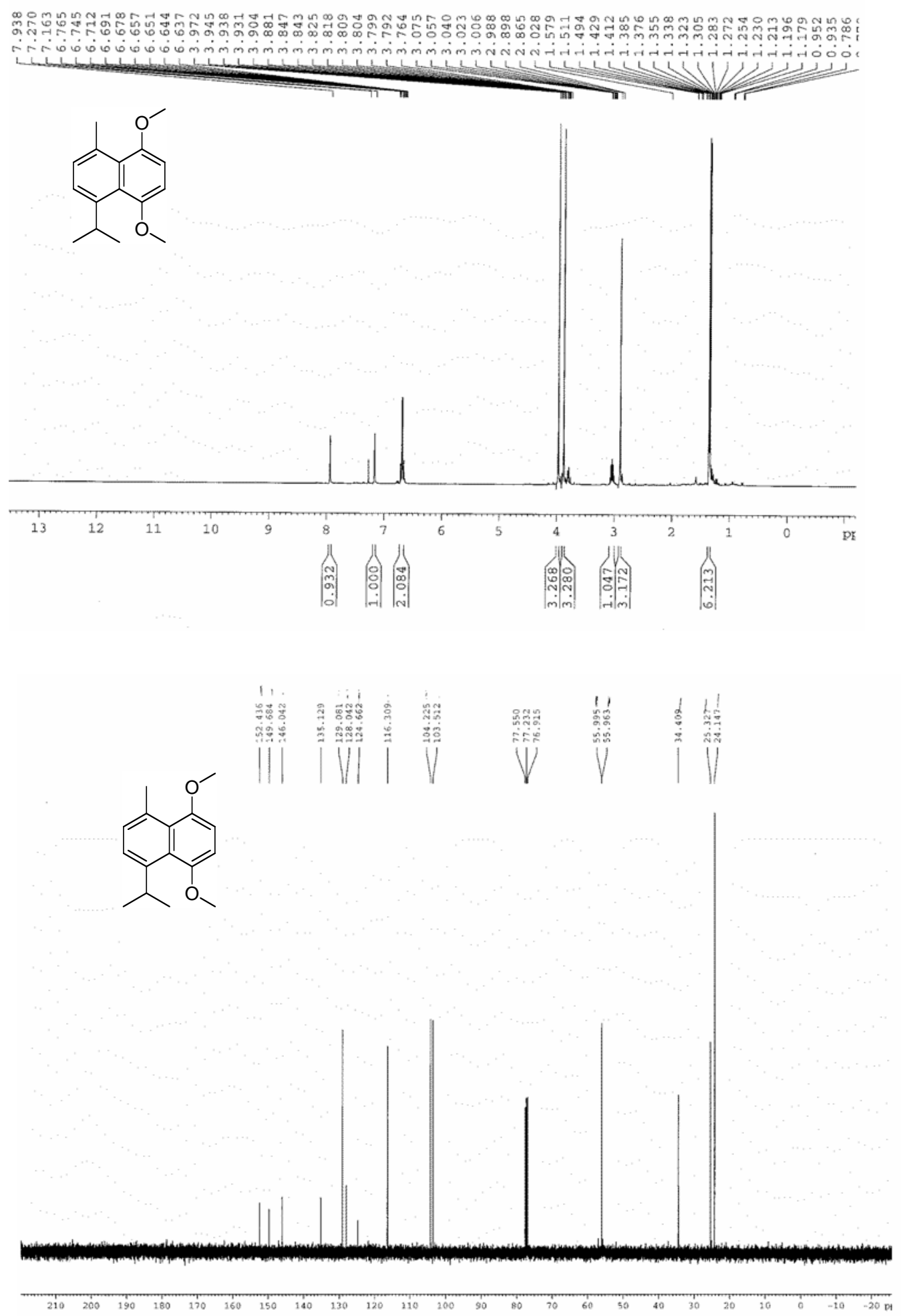

1- Isopropyl-5,8-dimethoxy-4-methylnaphthalene (11) 


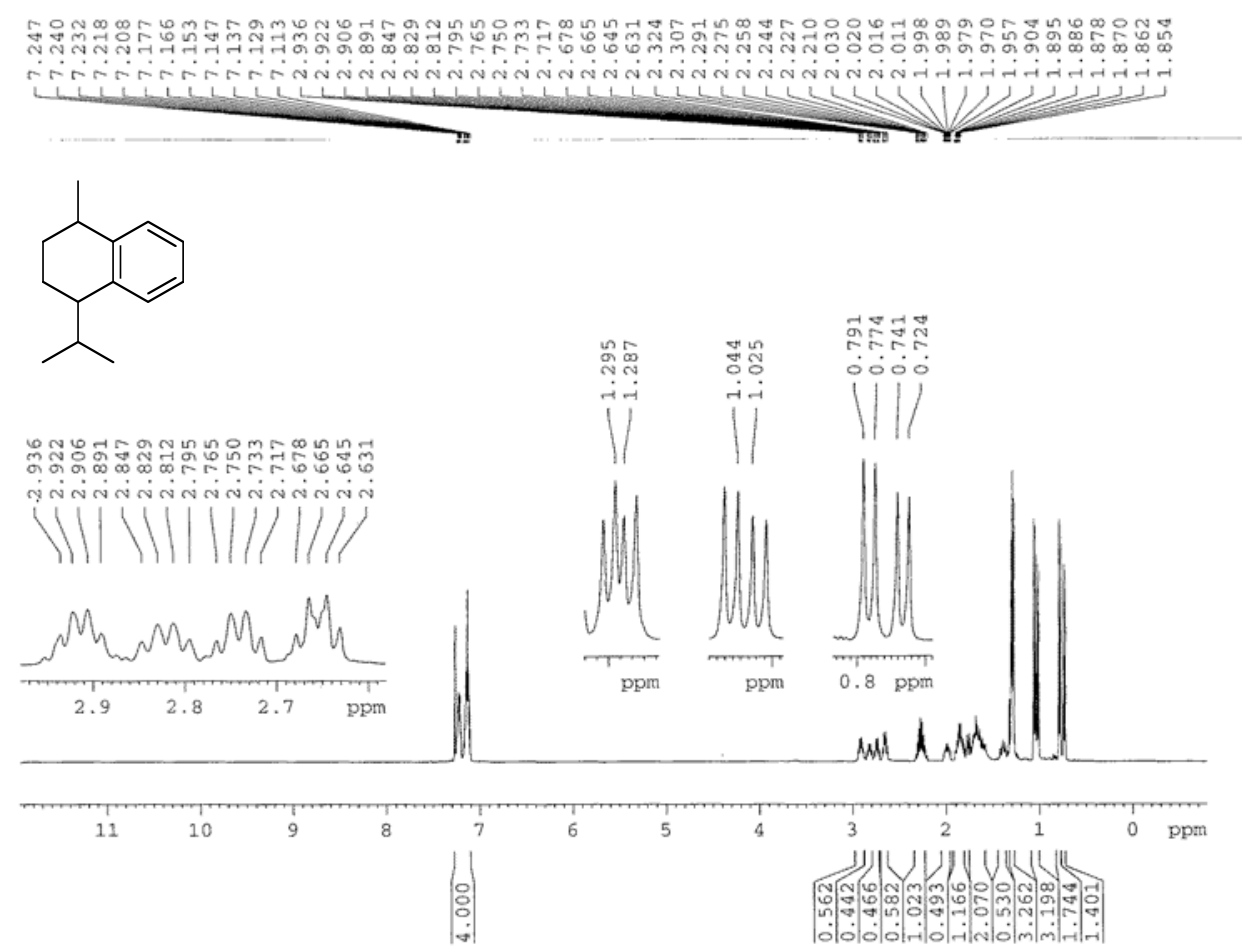

1-Isopropyl-4-methyl-1,2,3,4-tetrahydronaphthalene (13) 


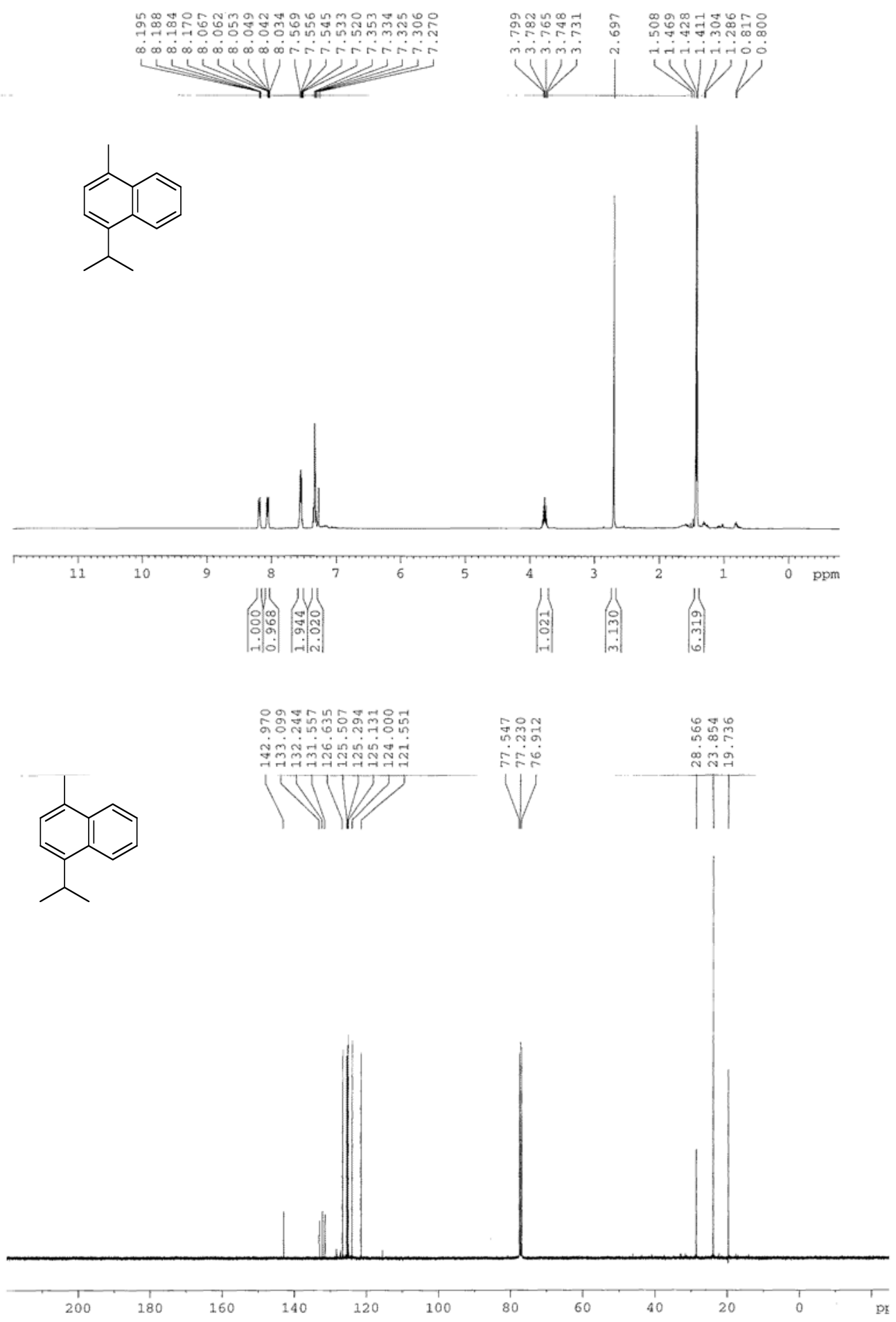

1-Isopropyl-4-methyl naphthalene (14) 


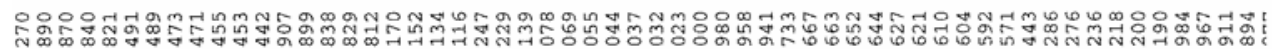
年

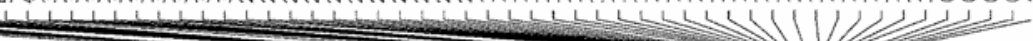<smiles>COc1c(C)ccc([C@@H](C)CCC=C(C)COC(C)=O)c1OC</smiles>

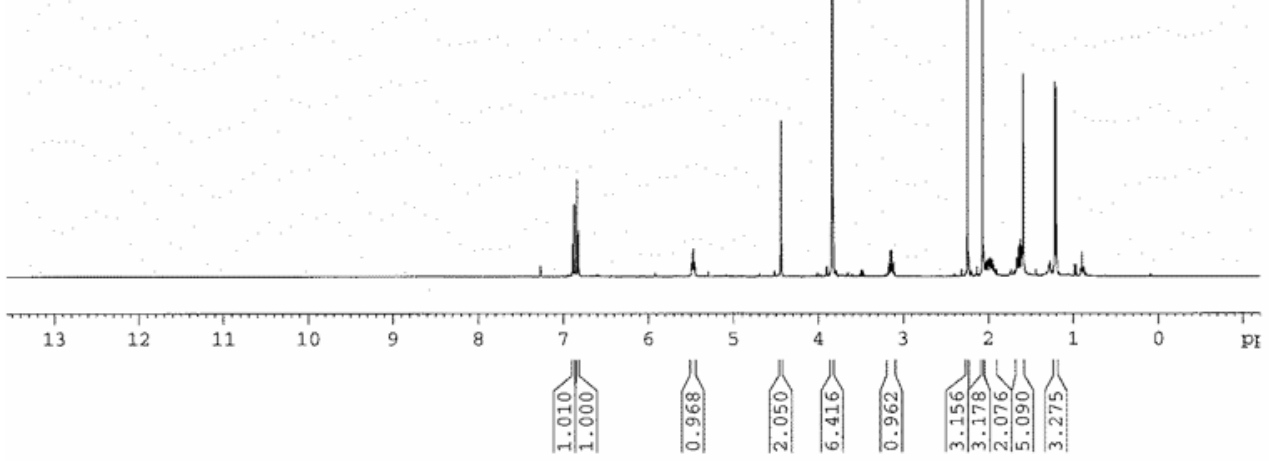<smiles>COc1c(C)ccc([C@@H](C)CC/C=C(/C)COC(C)=O)c1OC</smiles>
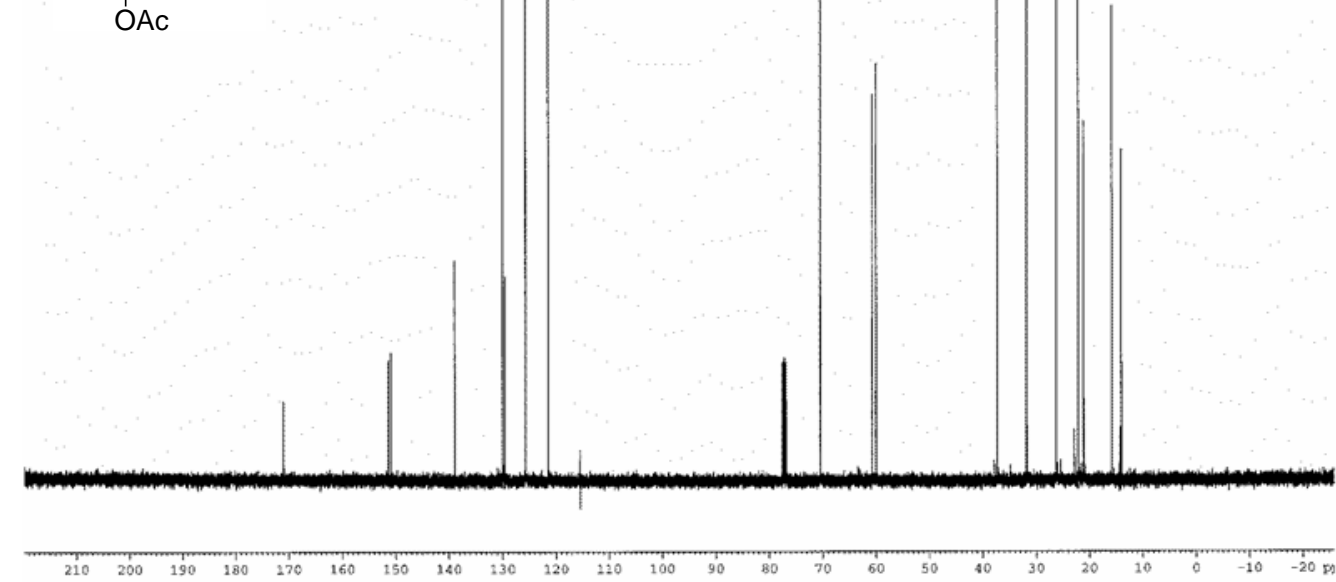

(2E)-6-(2,3-dimethoxy-4-methylphenyl)-2-methylhept-2-enyl acetate (17) 

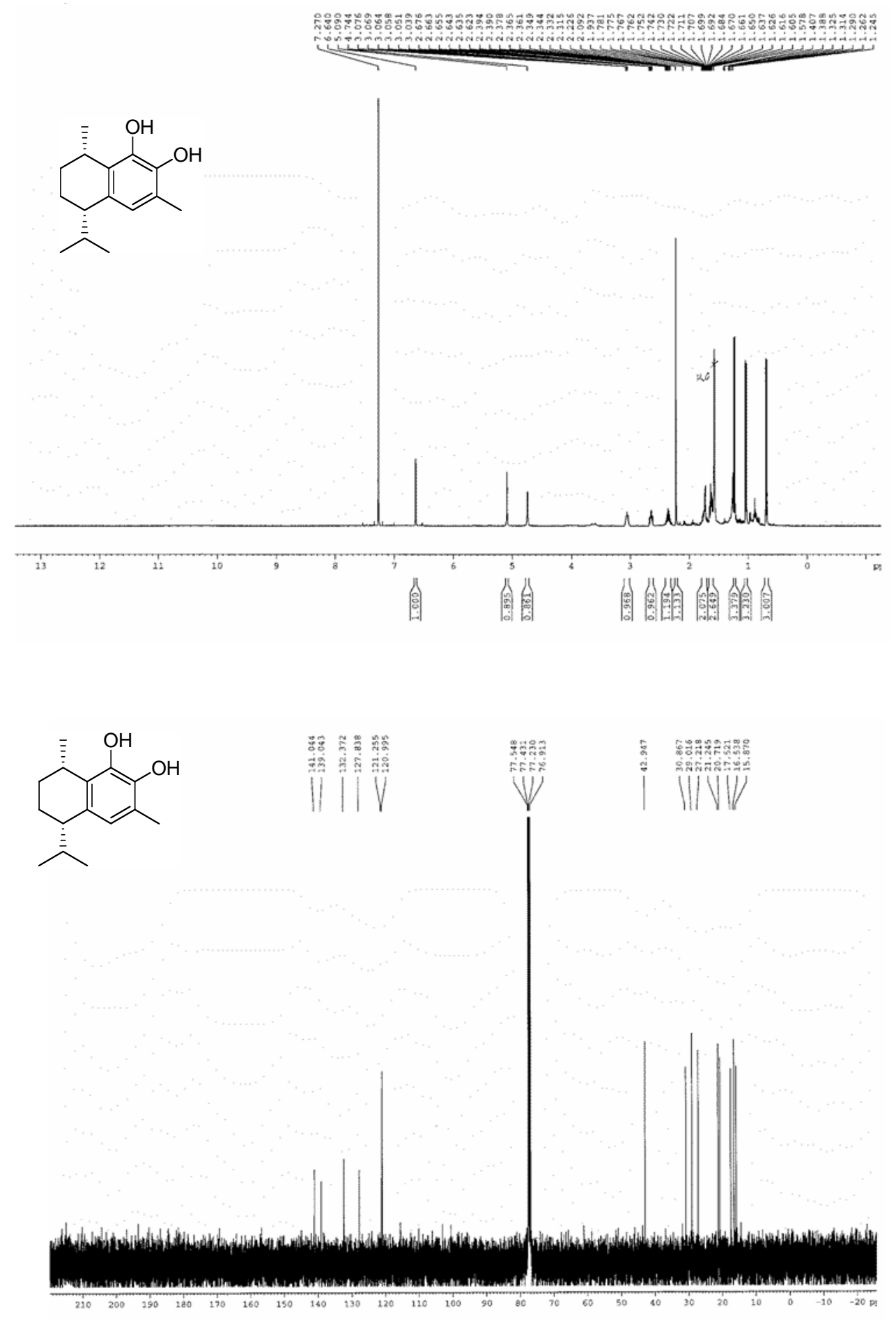

7,8-Dihydroxycalamenene (1a) 

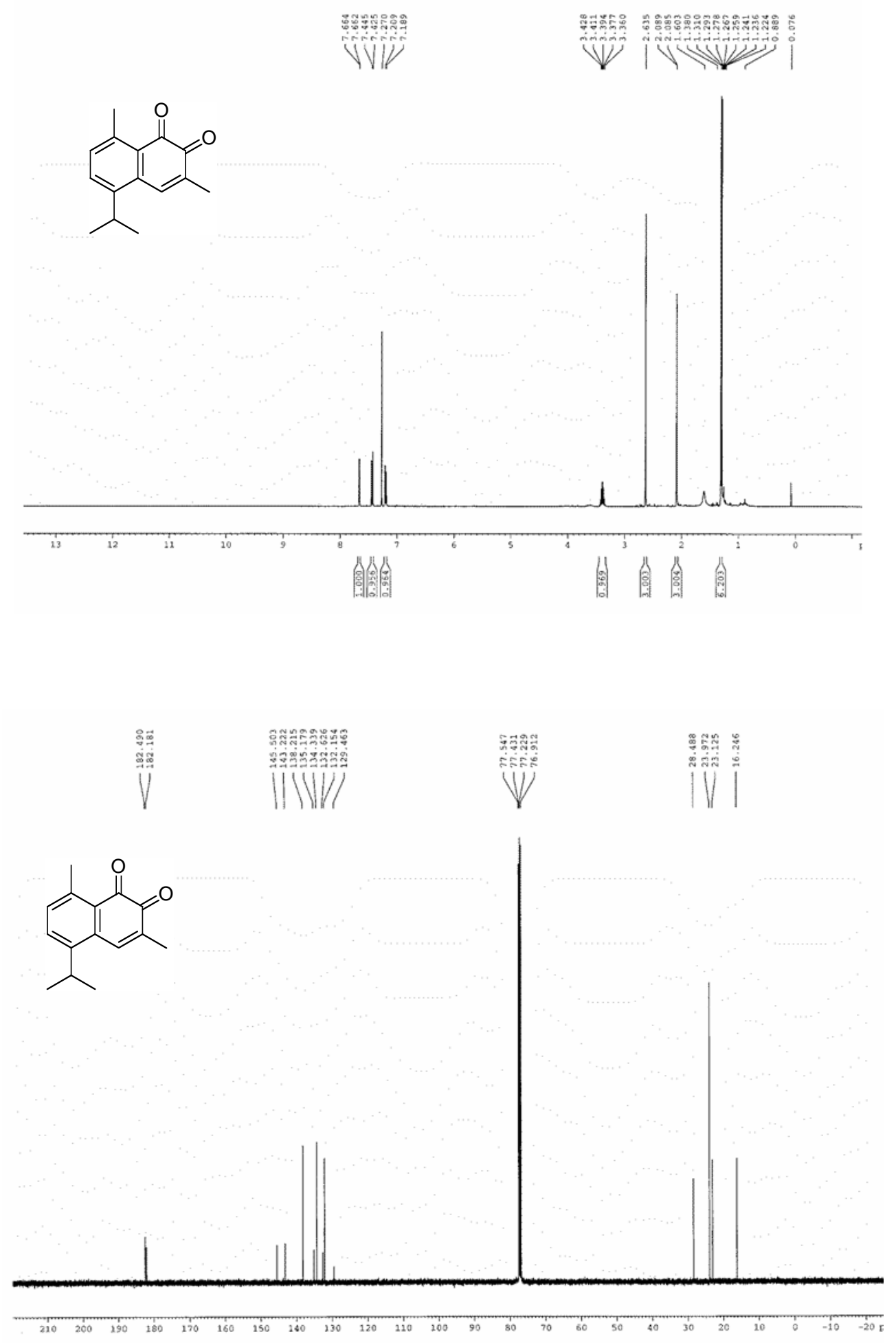

Mansonone C (2) 